\title{
OSCILLATORY BEHAVIOR OF SECOND ORDER DAMPED NEUTRAL DIFFERENTIAL EQUATIONS WITH DISTRIBUTED DEVIATING ARGUMENTS
}

\author{
SAID R. GRACE, JOHN R. GRAEF, AND ERCAN TUNÇ
}

Received 15 May, 2017

\begin{abstract}
The authors establish some new criteria for the oscillation of second order damped nonlinear neutral differential equations with distributed deviating arguments. Two examples are also provided to illustrate the results.

2010 Mathematics Subject Classification: 34C10; 34K11; 34K40

Keywords: Second order, neutral differential equations, damped equation, oscillation, asymptotic behavior, distributed deviating arguments
\end{abstract}

\section{INTRODUCTION}

This paper deals with oscillatory behavior of all solutions of the second order damped nonlinear neutral differential equation with distributed deviating arguments

$$
\begin{gathered}
\left(r(t)\left(y^{\prime}(t)\right)^{\alpha}\right)^{\prime}+p(t)\left(y^{\prime}(t)\right)^{\alpha}+\int_{a}^{b} q(t, \tau) f(t, x(g(t, \tau))) d \tau=0, \\
t \geq t_{0} \geq 0,
\end{gathered}
$$

where $y(t)=x(t)+b(t) \phi(x(\sigma(t))), 0<a<b$, and $\alpha$ is the quotient of odd positive integers.

In the remainder of the paper we assume that:

(i) $p, r:\left[t_{0}, \infty\right) \rightarrow \mathbb{R}^{+}$are continuous functions such that $r(t)$ is nondecreasing, and

$$
\int_{t_{0}}^{\infty}\left(\frac{1}{r(s)} A\left(s, t_{0}\right)\right)^{1 / \alpha} d s<\infty
$$

where

$$
A\left(t, t_{0}\right)=\exp \left(\int_{t_{0}}^{t} \frac{-p(s)}{r(s)} d s\right) ;
$$

(c) 2017 Miskolc University Press 
(ii) $b:\left[t_{0}, \infty\right) \rightarrow \mathbb{R}$ is a continuous function such that $0 \leq b(t)<1$;

(iii) $q:\left[t_{0}, \infty\right) \times[a, b] \rightarrow \mathbb{R}^{+}$is a continuous function;

(iv) $g:\left[t_{0}, \infty\right) \times[a, b] \rightarrow \mathbb{R}$ is a continuous function such that $g(t, \tau)$ is decreasing in $\tau, g(t, \tau) \leq t$, and $g(t, \tau) \rightarrow \infty$ as $t \rightarrow \infty, \tau \in[a, b]$;

(v) $f(t, u):\left[t_{0}, \infty\right) \times \mathbb{R} \rightarrow \mathbb{R}$ is a continuous function such that $u f(t, u)>0$ for all $u \neq 0$ and there exists a positive constant $\mu$ such that

$$
f(t, u) / u^{\alpha} \geq \mu \quad \text { for } u \neq 0
$$

(vi) $\phi: \mathbb{R} \rightarrow \mathbb{R}$ is a continuous function such that $u \phi(u)>0$ for all $u \neq 0$ and there exists a real number $\beta$ with $0<\beta<1$ such that $\phi(u) / u \leq \beta$ for $u \neq 0$;

(vii) $\sigma:\left[t_{0}, \infty\right) \rightarrow \mathbb{R}$ is a continuous function such that $\sigma(t) \leq t$, and $\sigma(t) \rightarrow \infty$ as $t \rightarrow \infty$.

By a solution of (1) we mean a function $x:\left[t_{x}, \infty\right) \rightarrow \mathbb{R}$ such that $y \in C^{1}\left(\left[t_{x}, \infty\right), \mathbb{R}\right)$ and $r(t)\left(y^{\prime}(t)\right)^{\alpha} \in C^{1}\left(\left[t_{x}, \infty\right), \mathbb{R}\right)$, and which satisfies equation (1) on $\left[t_{x}, \infty\right)$. Without further mention, we will assume throughout that every solution $x(t)$ of (1) under consideration here is continuable to the right and nontrivial, i.e., $x(t)$ is defined on some ray $\left[t_{x}, \infty\right)$, for some $t_{x} \geq t_{0}$, and $\sup \{|x(t)|: t \geq T\}>0$ for every $T \geq t_{x}$. Moreover, we tacitly assume that (1) possesses such solutions. Such a solution is said to be oscillatory if it has arbitrarily large zeros on $\left[t_{x}, \infty\right)$; otherwise it is called nonoscillatory. Equation (1) is said to be oscillatory if all its solutions are oscillatory.

In recent years, there has been much research activity concerning the oscillation and nonoscillation of solutions of various differential equations, and we refer the reader to the papers $[1,2,4-7,9,10,12,13]$ and the references therein as examples of recent results on this topic. However, oscillation results for second order differential equations with distributed deviating arguments are relatively scarce in the literature; some results can be found, for example, in $[3,8,11,14]$ and the references contained therein. Our purpose here is to establish some new oscillation criteria for equation (1) different from those in $[3,8,11,14]$ and to contribute to the growing body of research on second order neutral differential equations in general and those with distributed delays and a damping term in particular.

\section{MAIN RESULTS}

In this section, we establish some new criteria for the oscillation of equation (1). It will be convenient to employ the following notations.

$$
Q(t)=\int_{a}^{b} q(t, \tau) B^{\alpha}(g(t, \tau)) d \tau, \quad g(t, b)=g(t), \quad B(t)=1-\beta b(t),
$$




$$
Q^{*}(t)=\int_{a}^{b} q(t, \tau) Q_{1}^{\alpha}(g(t, \tau)) d \tau, \quad R(t)=\int_{t}^{\infty}\left(\frac{A(s, t)}{r(s)}\right)^{1 / \alpha} d s,
$$

and

$$
Q_{1}(t)=\left[1-\beta b(t)\left(\frac{R(\sigma(t))}{R(t)}\right)\right] .
$$

Throughout the paper we assume that

$$
Q_{1}(t) \geq 0 \text { for } t \geq t_{0} .
$$

We begin with the following lemma that will be used to prove our main results.

Lemma 1 ([4]). Let $\tau(t) \in C\left(\left[t_{0}, \infty\right), \mathbb{R}\right), \tau(t) \leq t$, and $\tau(t) \rightarrow \infty$ as $t \rightarrow \infty$. Assume $x \in C^{2}[T, \infty)$ satisfies

$$
x(t)>0, \quad x^{\prime}(t)>0, \quad \text { and } \quad x^{\prime \prime}(t) \leq 0 \quad \text { for } t \geq T \geq t_{0} .
$$

Then for each $k \in(0,1)$, there exists $T_{k} \geq T$ such that

$$
\frac{x(\tau(t))}{\tau(t)} \geq k \frac{x(t)}{t} \quad \text { for } t \geq T_{k} .
$$

Theorem 1. Let conditions (i)-(vii), (1.2), and (2.1) hold. If there exists a positive function $\rho(t) \in C^{1}\left(\left[t_{0}, \infty\right), \mathbb{R}\right)$ such that

$$
\begin{gathered}
p(t) \geq \frac{r(t) \rho^{\prime}(t)}{\rho(t)} \quad \text { for } t \geq t_{0}, \\
\int_{t_{0}}^{\infty} \rho(s) Q(s)\left(\frac{g(s)}{s}\right)^{\alpha} d s=\infty,
\end{gathered}
$$

and

$$
\int_{t_{0}}^{\infty}\left[\frac{A\left(t, t_{0}\right)}{r(t)} \int_{t_{0}}^{t} Q^{*}(s) R^{\alpha}(s) d s\right]^{1 / \alpha} d t=\infty,
$$

then equation (1) is oscillatory.

Proof. Let $x(t)$ be a nonoscillatory solution of (1), say $x(t)>0$ and $x(\sigma(t))>0$ for $t \in\left[t_{1}, \infty\right)$, and $x(g(t, \tau))>0$ for $(t, \tau) \in\left[t_{1}, \infty\right) \times[a, b]$ for some $t_{1} \in\left[t_{0}, \infty\right)$. From (1) and (v), we have

$$
\left(r(t)\left(y^{\prime}(t)\right)^{\alpha}\right)^{\prime}+p(t)\left(y^{\prime}(t)\right)^{\alpha}+\mu \int_{a}^{b} q(t, \tau) x^{\alpha}(g(t, \tau)) d \tau \leq 0 \quad \text { for } t \geq t_{1},
$$

so

$$
\left(r(t)\left(y^{\prime}(t)\right)^{\alpha}\right)^{\prime}+p(t)\left(y^{\prime}(t)\right)^{\alpha} \leq 0 .
$$


Setting $u(t)=r(t)\left(y^{\prime}(t)\right)^{\alpha}$, we see that

$$
u^{\prime}(t)+\frac{p(t)}{r(t)} u(t) \leq 0
$$

which implies

$$
\left(u(t) \exp \left(\int_{t_{1}}^{t} \frac{p(s)}{r(s)} d s\right)\right)^{\prime} \leq 0 .
$$

Thus, $u(t) \exp \left(\int_{t_{1}}^{t} \frac{p(s)}{r(s)} d s\right)$ is decreasing and so is eventually of one sign. Therefore, $y^{\prime}(t)$ eventually has a fixed sign say for $t \geq t_{2} \geq t_{1}$.

We shall then distinguish the following two cases:

Case (I): $y^{\prime}(t)>0$ for $t \geq t_{2}$;

Case (II): $y^{\prime}(t)<0$ for $t \geq t_{2}$.

Consider Case (I). From (1) and (v), we obtain

$$
\left(r(t)\left(y^{\prime}(t)\right)^{\alpha}\right)^{\prime}+p(t)\left(y^{\prime}(t)\right)^{\alpha}+\mu \int_{a}^{b} q(t, \tau) x^{\alpha}(g(t, \tau)) d \tau \leq 0 \quad \text { for } t \geq t_{1} .
$$

Since $x(t) \leq y(t)$, in view of (vi) and (vii), we have

$$
y(t) \leq x(t)+\beta b(t) x(\sigma(t)) \leq x(t)+\beta b(t) y(\sigma(t)) \leq x(t)+\beta b(t) y(t),
$$

from which we see that

$$
x(t) \geq(1-\beta b(t)) y(t)=B(t) y(t) .
$$

Using (2.8) in (2.7) gives

$$
\left(r(t)\left(y^{\prime}(t)\right)^{\alpha}\right)^{\prime}+p(t)\left(y^{\prime}(t)\right)^{\alpha}+\mu \int_{a}^{b} q(t, \tau) B^{\alpha}(g(t, \tau)) y^{\alpha}(g(t, \tau)) d \tau \leq 0 .
$$

In view of (iv) and the fact that $y(t)$ is increasing, the last inequality takes the form

$$
\left(r(t)\left(y^{\prime}(t)\right)^{\alpha}\right)^{\prime}+p(t)\left(y^{\prime}(t)\right)^{\alpha}+\mu Q(t) y^{\alpha}(g(t)) \leq 0 .
$$

From (2.6) we see that

$$
r^{\prime}(t)\left(y^{\prime}(t)\right)^{\alpha}+\alpha r(t)\left(y^{\prime}(t)\right)^{\alpha-1} y^{\prime \prime}(t)+p(t)\left(y^{\prime}(t)\right)^{\alpha} \leq 0 .
$$

Since $r(t)$ is positive and nondecreasing, this implies $y^{\prime \prime}(t)<0$. Therefore, by Lemma 1 , for $k \in(0,1)$ fixed, there exits $t_{3} \geq t_{2}$ such that

$$
\frac{y(g(t))}{g(t)} \geq k \frac{y(t)}{t} \quad \text { for all } t \geq t_{3} .
$$


Substituting (2.10) into (2.9) gives

$$
\left(r(t)\left(y^{\prime}(t)\right)^{\alpha}\right)^{\prime}+p(t)\left(y^{\prime}(t)\right)^{\alpha}+\mu k^{\alpha} Q(t)\left(\frac{g(t)}{t}\right)^{\alpha} y^{\alpha}(t) \leq 0 \quad \text { for } t \geq t_{3} .
$$

Now define the function $w(t)$ by

$$
w(t)=\rho(t) \frac{r(t)\left(y^{\prime}(t)\right)^{\alpha}}{y^{\alpha}(t)} \quad \text { for } t \geq t_{3} .
$$

Clearly, $w(t)>0$, and

$$
w^{\prime}(t)=\frac{\rho^{\prime}(t)}{\rho(t)} w(t)+\rho(t) \frac{\left(r(t)\left(y^{\prime}(t)\right)^{\alpha}\right)^{\prime}}{y^{\alpha}(t)}-\rho(t) \frac{r(t)\left(y^{\prime}(t)\right)^{\alpha}\left(y^{\alpha}(t)\right)^{\prime}}{y^{2 \alpha}(t)} .
$$

Using (2.11) and (2.12) in (2.13), we obtain

$$
w^{\prime}(t) \leq-\mu k^{\alpha} \rho(t) Q(t)\left(\frac{g(t)}{t}\right)^{\alpha}+\left(\frac{\rho^{\prime}(t)}{\rho(t)}-\frac{p(t)}{r(t)}\right) w(t)-\frac{\alpha(w(t))^{1+1 / \alpha}}{(r(t) \rho(t))^{1 / \alpha}} .
$$

In view of (2.3) and $w(t)>0$, the last inequality implies

$$
w^{\prime}(t) \leq-\mu k^{\alpha} \rho(t) Q(t)\left(\frac{g(t)}{t}\right)^{\alpha} \text { for } t \geq t_{3} .
$$

Integrating this inequality from $t_{3}$ to $t$ yields

$$
0 \leq w(t) \leq w\left(t_{3}\right)-\mu k^{\alpha} \int_{t_{3}}^{t} \rho(s) Q(s)\left(\frac{g(s)}{s}\right)^{\alpha} d s \rightarrow-\infty
$$

as $t \rightarrow \infty$, which is a contradiction.

Next, we consider Case (II). From (1.1), we obtain

$$
\begin{aligned}
\left(\frac{r(t)\left(y^{\prime}(t)\right)^{\alpha}}{A\left(t, t_{0}\right)}\right)^{\prime} & =\frac{\left(r(t)\left(y^{\prime}(t)\right)^{\alpha}\right)^{\prime}}{A\left(t, t_{0}\right)}-\frac{r(t)\left(y^{\prime}(t)\right)^{\alpha} A^{\prime}\left(t, t_{0}\right)}{A^{2}\left(t, t_{0}\right)} \\
& =\frac{1}{A\left(t, t_{0}\right)}\left[\left(r(t)\left(y^{\prime}(t)\right)^{\alpha}\right)^{\prime}+p(t)\left(y^{\prime}(t)\right)^{\alpha}\right] \\
& =-\frac{1}{A\left(t, t_{0}\right)} \int_{a}^{b} q(t, \tau) f(t, x(g(t, \tau))) d \tau \leq 0,
\end{aligned}
$$

i.e, $r(t)\left(y^{\prime}(t)\right)^{\alpha} / A\left(t, t_{0}\right)$ is decreasing. Hence, we have

$$
\frac{r(s)\left(y^{\prime}(s)\right)^{\alpha}}{A\left(s, t_{0}\right)} \leq \frac{r(t)\left(y^{\prime}(t)\right)^{\alpha}}{A\left(t, t_{0}\right)} \quad \text { for } s \geq t \geq t_{3}
$$


from which we obtain

$$
y^{\prime}(s) \leq r^{1 / \alpha}(t) y^{\prime}(t)\left(\frac{A(s, t)}{r(s)}\right)^{1 / \alpha} \quad \text { for } s \geq t .
$$

It follows that

$$
y(u)-y(t) \leq r^{1 / \alpha}(t) y^{\prime}(t) \int_{t}^{u}\left(\frac{A(s, t)}{r(s)}\right)^{1 / \alpha} d s .
$$

Letting $u \rightarrow \infty$ in the last inequality, we see that

$$
y(t) \geq-r^{1 / \alpha}(t) y^{\prime}(t) \int_{t}^{\infty}\left(\frac{A(s, t)}{r(s)}\right)^{1 / \alpha} d s=R(t)\left(-r^{1 / \alpha}(t) y^{\prime}(t)\right),
$$

which implies

$$
\left(\frac{y(t)}{R(t)}\right)^{\prime} \geq 0,
$$

and hence $y(t) / R(t)$ is nondecreasing. From this, the definition of $y(t)$, (vi), and (vii), we have

$$
\begin{aligned}
x(t) & \geq y(t)-\beta b(t) x(\sigma(t)) \geq y(t)-\beta b(t) y(\sigma(t) \\
& \geq\left[1-\beta b(t)\left(\frac{R(\sigma(t))}{R(t)}\right)\right] y(t)=Q_{1}(t) y(t) .
\end{aligned}
$$

Again using the fact that $r(t)\left(y^{\prime}(t)\right)^{\alpha} / A\left(t, t_{0}\right)$ is decreasing, we have

$$
r(t)\left(-y^{\prime}(t)\right)^{\alpha} \geq \frac{A\left(t, t_{0}\right)}{A\left(t_{3}, t_{0}\right)} r\left(t_{2}\right)\left(-y^{\prime}\left(t_{2}\right)\right)^{\alpha}=\gamma A\left(t, t_{0}\right)>0 \quad \text { for } t \geq t_{3},
$$

for some positive constant $\gamma$.

Combining (2.17) and (2.15) gives

$$
y(t) \geq \gamma^{1 / \alpha} R(t) A^{1 / \alpha}\left(t, t_{0}\right) \quad \text { for } t \geq t_{3} .
$$

From (2.7), (2.16), (iv), and the fact that $y(t)$ is decreasing, we obtain

$$
\begin{aligned}
-\left(r(t)\left(y^{\prime}(t)\right)^{\alpha}\right)^{\prime} & \geq p(t)\left(y^{\prime}(t)\right)^{\alpha}+\mu \int_{a}^{b} q(t, \tau) x^{\alpha}(g(t, \tau)) d \tau \\
& \geq p(t)\left(y^{\prime}(t)\right)^{\alpha}+\mu \int_{a}^{b} q(t, \tau) Q_{1}^{\alpha}(g(t, \tau)) y^{\alpha}(g(t, \tau)) d \tau \\
& \geq p(t)\left(y^{\prime}(t)\right)^{\alpha}+\mu \int_{a}^{b} q(t, \tau) Q_{1}^{\alpha}(g(t, \tau)) y^{\alpha}(t) d \tau
\end{aligned}
$$




$$
=p(t)\left(y^{\prime}(t)\right)^{\alpha}+\mu Q^{*}(t) y^{\alpha}(t) .
$$

Using (2.18) in (2.19), we arrive at

$$
-\left(r(t)\left(y^{\prime}(t)\right)^{\alpha}\right)^{\prime} \geq p(t)\left(y^{\prime}(t)\right)^{\alpha}+\mu \gamma Q^{*}(t) A\left(t, t_{0}\right) R^{\alpha}(t) .
$$

With $U(t)=r(t)\left(y^{\prime}(t)\right)^{\alpha},(2.20)$ becomes

$$
U^{\prime}(t) \leq-\frac{p(t)}{r(t)} U(t)-\mu \gamma Q^{*}(t) A\left(t, t_{0}\right) R^{\alpha}(t),
$$

which can be written as

$$
\left(\frac{U(t)}{A\left(t, t_{0}\right)}\right)^{\prime} \leq-\mu \gamma Q^{*}(t) R^{\alpha}(t), \quad \text { for } t \geq t_{3} .
$$

Integrating this inequality from $t_{3}$ to $t$ gives

$$
\begin{aligned}
U(t) & \leq U\left(t_{3}\right) A\left(t, t_{3}\right)-\mu \gamma A\left(t, t_{0}\right) \int_{t_{3}}^{t} Q^{*}(s) R^{\alpha}(s) d s \\
& \leq-\mu \gamma A\left(t, t_{0}\right) \int_{t_{3}}^{t} Q^{*}(s) R^{\alpha}(s) d s,
\end{aligned}
$$

which leads to

$$
y^{\prime}(t) \leq-(\mu \gamma)^{1 / \alpha}\left(\frac{A\left(t, t_{0}\right)}{r(t)} \int_{t_{3}}^{t} Q^{*}(s) R^{\alpha}(s) d s\right)^{1 / \alpha} .
$$

An integration of the last inequality from $t_{3}$ to $t$ implies

$$
0<y(t) \leq y\left(t_{3}\right)-(\mu \gamma)^{1 / \alpha} \int_{t_{3}}^{t}\left(\frac{A\left(u, t_{0}\right)}{r(u)} \int_{t_{3}}^{u} Q^{*}(s) R^{\alpha}(s) d s\right)^{1 / \alpha} d u \rightarrow-\infty
$$

as $t \rightarrow \infty$, by (2.5). This is contradicts the fact that $y(t)>0$ and completes the proof of the theorem.

Our next theorem gives conditions under which a solution will either oscillate or converge to zero as $t \rightarrow \infty$.

Theorem 2. In Theorem 2.2, if condition (2.5) is replaced by

$$
\int_{t_{0}}^{\infty}\left(\frac{A\left(t, t_{0}\right)}{r(t)}\right)^{1 / \alpha}\left(\int_{t_{0}}^{t} Q^{*}(s) d s\right)^{1 / \alpha} d t=\infty
$$

then any solution $x$ of equation (1) either oscillates or converges to zero as $t \rightarrow \infty$. 
Proof. Let $x(t)$ be a nonoscillatory solution of (1), say $x(t)>0, x(\sigma(t))>0$ for $t \in\left[t_{1}, \infty\right)$, and $x(g(t, \tau))>0$ for $(t, \tau) \in\left[t_{1}, \infty\right) \times[a, b]$ for some $t_{1} \in\left[t_{0}, \infty\right)$. We again distinguish the two cases:

$$
\text { (I) } y^{\prime}(t)>0 \quad \text { or } \quad \text { (II) } y^{\prime}(t)<0
$$

for $t \geq t_{2}$ for some $t_{2} \geq t_{1}$.

The proof if Case (I) holds is similar to that of Case (I) in the proof of Theorem 1, and hence is omitted.

Next, we consider Case (II). Since $y(t)>0$ and $y^{\prime}(t)<0$, there exists a constant $c$ such that

$$
\lim _{t \rightarrow \infty} y(t)=c<\infty
$$

where $c \geq 0$.

If $c>0$, there exists $t_{3} \geq t_{2}$ such that

$$
y(t) \geq c \quad \text { for } t \geq t_{3} .
$$

From (2.7), (2.16), (2.22), and (iv), we see that

$$
\begin{aligned}
-\left(r(t)\left(y^{\prime}(t)\right)^{\alpha}\right)^{\prime} & \geq p(t)\left(y^{\prime}(t)\right)^{\alpha}+\mu \int_{a}^{b} q(t, \tau) x^{\alpha}(g(t, \tau)) d \tau \\
& \geq p(t)\left(y^{\prime}(t)\right)^{\alpha}+\mu \int_{a}^{b} q(t, \tau) Q_{1}^{\alpha}(g(t, \tau)) y^{\alpha}(g(t, \tau)) d \tau \\
& \left.\geq p(t)\left(y^{\prime}(t)\right)^{\alpha}+\mu \int_{a}^{b} q(t, \tau) Q_{1}^{\alpha}(g(t, \tau)) y^{\alpha}(t)\right) d \tau \\
& \geq p(t)\left(y^{\prime}(t)\right)^{\alpha}+\mu c^{\alpha} Q^{*}(t),
\end{aligned}
$$

which can be written as

$$
\begin{aligned}
\left(\frac{\left(r(t)\left(y^{\prime}(t)\right)^{\alpha}\right)}{A\left(t, t_{0}\right)}\right)^{\prime} & \leq-\mu c^{\alpha} \frac{1}{A\left(t, t_{0}\right)} Q^{*}(t) \\
& \leq-\mu c^{\alpha} Q^{*}(t), \quad \text { for } t \geq t_{3} .
\end{aligned}
$$

Integrating (2.23) from $t_{3}$ to $t$, we obtain

$$
\frac{r(t)\left(y^{\prime}(t)\right)^{\alpha}}{A\left(t, t_{0}\right)} \leq \frac{r\left(t_{3}\right)\left(y^{\prime}\left(t_{3}\right)\right)^{\alpha}}{A\left(t_{3}, t_{0}\right)}-\mu c^{\alpha} \int_{t_{3}}^{t} Q^{*}(s) d s \leq-\mu c^{\alpha} \int_{t_{3}}^{t} Q^{*}(s) d s,
$$


which leads to

$$
y^{\prime}(t) \leq-c \mu^{1 / \alpha}\left(\frac{A\left(t, t_{0}\right)}{r(t)}\right)^{1 / \alpha}\left(\int_{t_{3}}^{t} Q^{*}(s) d s\right)^{1 / \alpha} .
$$

Integrating from $t_{3}$ to $u \geq t_{3}$ and applying (2.21), we see that

$$
0 \leq y(u) \leq y\left(t_{3}\right)-c \mu^{1 / \alpha} \int_{t_{3}}^{u}\left(\frac{A\left(t, t_{0}\right)}{r(t)}\right)^{1 / \alpha}\left(\int_{t_{3}}^{t} Q^{*}(s) d s\right)^{1 / \alpha} d t \rightarrow-\infty
$$

as $u \rightarrow \infty$ which is a contradiction. Hence, we have $c=0$, that is, $\lim _{t \rightarrow \infty} y(t)=0$. Since $0<x(t) \leq y(t)$ on $\left[t_{1}, \infty\right)$, we have $\lim _{t \rightarrow \infty} x(t)=0$, and this completes the proof of the theorem.

\section{DISCUSSION AND EXAMPLES}

The results here appear to be one of the first attempts to look at equations in the form of (1) containing a damping term. We hope that this will encourage other researchers to explore similar problems. We conclude this paper with two examples to illustrate the applicability of our results.

Example 1. Consider the neutral differential equation

$$
\begin{gathered}
\left(t^{2}\left(y^{\prime}(t)\right)^{1 / 3}\right)^{\prime}+t\left(y^{\prime}(t)\right)^{1 / 3}+\int_{1}^{2}\left(t^{6}+2 \tau\right) f(t, x(t / 2+1 / 3 \tau)) d \tau=0, \\
t \geq 1 .
\end{gathered}
$$

Here we have $\alpha=1 / 3, r(t)=t^{2}, p(t)=t, q(t, \tau)=t^{6}+2 \tau, g(t, \tau)=t / 2+$ $1 / 3 \tau, a=1, b=2$, and we take $y(t)=x(t)+\frac{1}{32} \phi(x(t / 2))$ with $\phi(u)=\frac{1}{2} u$, and $f(t, u)=u^{\alpha}$. Then, $\beta=1 / 2, \mu=1, b(t)=1 / 32, B(t)=63 / 64, Q(t)=$ $(63 / 64)^{1 / 3}\left(t^{6}+3\right), Q^{*}(t)=(1 / 2)^{1 / 3}\left[t^{6}+3\right], Q_{1}(t)=1 / 2, A\left(t, t_{0}\right)=1 / t, A(s, t)=$ $t / s$, and $R(t)=1 / 8 t^{5}, R(\sigma(t))=4 / t^{5}$. It is clear that conditions (i)-(vii) and (1.2) hold. With $\rho(t)=t$, we see that condition (2.3) holds, and conditions (2.4) and (2.5) become

$$
(63 / 64)^{1 / 3} \int_{1}^{\infty} s\left(s^{6}+3\right)\left(\frac{s / 2+1 / 6}{s}\right)^{1 / 3} \geq(63 / 128)^{1 / 3} \int_{1}^{\infty} s^{7} d s=\infty,
$$

and

$$
\int_{1}^{\infty}\left[\frac{1}{t^{3}} \int_{1}^{t}\left(\frac{1}{2}\right)^{1 / 3}\left(s^{6}+3\right)\left(\frac{1}{8 s^{5}}\right)^{1 / 3} d s\right]^{3} d t
$$




$$
\geq\left(3^{3} / 2^{16}\right) \int_{1}^{\infty}\left[\frac{\left(t^{16 / 3}-1\right)}{t^{3}}\right]^{3} d t=\infty
$$

respectively, so equation (1) is oscillatory by Theorem 1 .

Example 2. In Example 1, if we take $q(t, \tau)=t^{5 / 2}+2 \tau$ instead of $q(t, \tau)=$ $t^{6}+2 \tau$, then we see that all conditions of Theorem 2 hold. Since condition (2.5) is not satisfied, Theorem 1 can not be applied to Example 2. In this case, any solution $x(t)$ of the equation either oscillates or converges to zero as $t \rightarrow \infty$.

\section{REFERENCES}

[1] R. P. Agarwal, S. R. Grace, and D. O'Regan, "On nonoscillatory solutions of differential inclusions," Amer. Math. Soc., vol. 131, pp. 129-140, 2002.

[2] R. P. Agarwal, S. R. Grace, and D. O'Regan, "Oscillation criteria for sublinear and superlinear second order differential inclusions," Mem. Differential Equations Math. Phys., vol. 28, pp. 1-12, 2003.

[3] T. Candan, "Oscillation of second-order nonlinear neutral dynamic equations on time scales with distributed deviating arguments," Comput. Math. Appl., vol. 62, pp. 4118-4125, 2011.

[4] L. Erbe, "Oscillation criteria for second order nonlinear delay equations," Canad. Math. Bull., vol. 16, pp. 49-56, 1973.

[5] L. Erbe, T. S. Hassan, and A. Peterson, "Oscillation criteria for nonlinear functional neutral dynamic equations on time scales," J. Difference Equ. Appl., pp. 1097-1116, 2009.

[6] S. R. Grace, R. P. Agarwal, M. Bohner, and D. O'Regan, "Oscillation of second-order strongly superlinear and strongly sublinear dynamic equations," Commun. Nonlinear Sci. Numer. Simulat., vol. 14, pp. 3463-3471, 2009.

[7] S. R. Grace, R. P. Agarwal, and D. O'Regan, "A selection of oscillation criteria for second-order differential inclusions," Appl. Math. Lett., vol. 22, pp. 153-158, 2009.

[8] S. R. Grace, E. Akın, and C. M. Dikmen, "On the oscillation of second order nonlinear neutral dynamic equations with distributed deviating arguments on time-scales," Dynam. Systems Appl., vol. 23, pp. 735-748, 2014.

[9] S. R. Grace, J. R. Graef, and E. Tunç, "Oscillatory behavior of a third-order neutral dynamic equation with distributed delays," Electron. J. Qual. Theory Differ. Equ., (Proc. 10th Coll. Qualitative Theory of Diff. Equ. (July 1-4, 2015, Szeged, Hungary), vol. 2016, no. 14, pp. 1-14, 2016.

[10] T. Li, Y. V. Rogovchenko, and C. Zhang, "Oscillation results for second-order nonlinear neutral differential equations," Adv. Difference Equ., vol. 2013, no. 336, p. 13pp, 2013.

[11] T. Li and E. Thandapani, "Oscillation of second-order quasi-linear neutral functional dynamic equations with distributed deviating arguments," J. Nonlinear Sci. Appl., vol. 4, pp. 180-192, 2011.

[12] T. Li, E. Thandapani, J. R. Graef, and E. Tunc, "Oscillation of second-order emden-fowler neutral differential equations," Nonlinear Stud., vol. 20, pp. 1-8, 2013.

[13] S. H. Saker, "Oscillation of second order neutral delay differential equations of emden-fowler type," Acta. Math. Hungar., vol. 100, pp. 37-62, 2003.

[14] E. Tunc and J. R. Graef, "Oscillation results for second order neutral dynamic equations with distributed deviating arguments,” Dynam. Systems Appl., vol. 23, pp. 289-303, 2014. 
Authors' addresses

\section{Said R. Grace}

Cairo University, Department of Engineering Mathematics, Faculty of En- gineering, Orman, Giza 12221, Egypt

E-mail address: saidgrace@yahoo.com

\section{John R. Graef}

Department of Mathematics, University of Tennessee at Chattanooga, Chattanooga, TN 37403, USA

E-mail address: John-Graef@utc.edu

\section{Ercan Tunç}

Gaziosmanpasa University, Department of Mathematics, Faculty of Arts and Sciences, 60240, Tokat, Turkey

E-mail address: ercantunc72@yahoo.com 